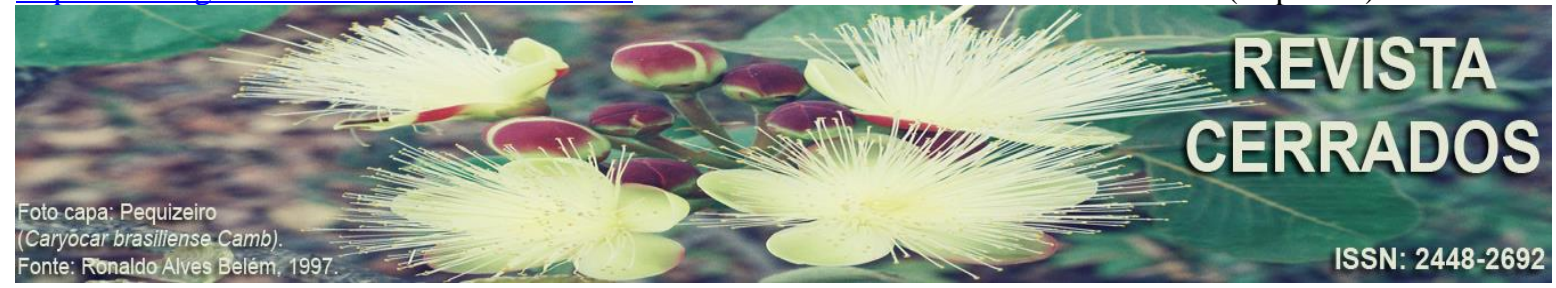

\title{
A AGENDA 2030 E O SANEAMENTO COMO INDICADOR DE SUSTENTABILIDADE ${ }^{1}$
}

\section{AGENDA AND THE SANITATION AS AN INDICATOR OF SUSTAINABILITY}

\section{LA AGENDA 2030 Y EL SANEAMIENTO COMO INDICADOR DE SOSTENIBILIDAD}

\author{
Duarcides Ferreira Mariosa \\ Pontifícia Universidade Católica de Campinas - PUC/Campinas, Campinas, \\ São Paulo, Brasil. \\ E-mail: 〈duarcidesmariosa@puc-campinas.edu.br>. \\ Samuel Carvalho De Benedicto \\ Pontifícia Universidade Católica de Campinas - PUC/Campinas, Campinas, \\ São Paulo, Brasil. \\ E-mail: 〈samuel.benedicto@puc-campinas.edu.br>. \\ Marcos Ricardo Rosa Georges \\ Pontifícia Universidade Católica de Campinas - PUC/Campinas, Campinas, \\ São Paulo, Brasil. \\ E-mail: 〈marcos.georges@puc-campinas.edu.br>. \\ Cibele Roberta Sugahara \\ Pontifícia Universidade Católica de Campinas - PUC/Campinas, Campinas, \\ São Paulo, Brasil. \\ E-mail: <cibelesu@ puc-campinas.edu.br>.
}

\section{RESUMO}

$\mathrm{O}$ acesso ao fornecimento de água potável e às condições adequadas de saneamento faz parte da Agenda 2030 para o Desenvolvimento Sustentável. Visto como medida higienizadora do ambiente, capaz de torná-lo salubre, atendendo às necessidades humanas, o saneamento é um serviço que se presta a um público, a um coletivo, independente da natureza jurídica de sua

\footnotetext{
${ }^{1}$ Discussão ajustada e ampliada do artigo publicado no "V Simpósio Internacional de Gestão de Projetos, Inovação e Sustentabilidade (V SINGEP) realizado em novembro de 2016 em São Paulo - SP.
} 
organização. O objetivo do presente estudo é identificar o comportamento das principais variáveis que compõem o item saneamento em uma comunidade ribeirinha localizada em área ambientalmente protegida na Amazônia brasileira. Trata-se de estudo longitudinal, de natureza descritiva e ecológica, baseado em pesquisa de campo, com aplicação de questionários estruturados e observação direta. As condições de saneamento encontradas mostraram-se precárias ou minimamente garantidas. O uso disseminado de "fossas negras" para lançamento do esgoto e a dificuldade no abastecimento de água potável mostraram-se preocupantes, indicando a necessidade de alguma forma de intervenção que possa corrigir essa tendência. Conclui-se que a análise das condições de saneamento da comunidade, examinadas em escala temporal, permite a observação do comportamento das variáveis que compõem o indicador utilizado e, dessa forma, a detecção de riscos ou vulnerabilidades que podem, ao persistirem as tendências negativas encontradas, comprometer aspectos econômicos, sociais e ambientais que caracterizam a sustentabilidade daquelas comunidades.

Palavras-chave: Sustentabilidade. Saneamento. Gestão em Saúde. Unidades de Conservação. Comunidades Ribeirinhas.

\begin{abstract}
The access to drinking water supply and adequate sanitation is part of Agenda 2030 for the Sustainable Development. Seen as a sanitary measure of the environment, able to make it healthy, sanitation is a service that lends itself to a public, regardless the legal nature of its organization. The objective of the present study is to identify the main variables that make up the sanitation item in a riverine community located in an environmentally protected area in the Brazilian Amazon. It is a longitudinal study, of a descriptive and ecological nature, based on field research with the application of structured questionnaires and direct observation. The sanitation conditions found were precarious or minimally guaranteed. The widespread use of "sewage cesspits" and the difficulty in supplying drinking water was worrisome, indicating the need for some form of intervention that could correct this trend. It is concluded that the analysis of community sanitation conditions, examined in a temporal scale, allows the observation of the behavior of the variables that make up the indicator used and, in this way, the detection of risks or vulnerabilities that can, with the persistence of negative trends economic, social and environmental aspects that characterize the sustainability of these communities.
\end{abstract}

Keywords: Sustainability. Sanitation. Health Management. Conservation units. Coastal Communities.

\title{
RESUMEN
}

El acceso al agua potable y al saneamiento adecuado es parte de la Agenda 2030 para el Desarrollo Sostenible. Visto como una medida desinfectante del medio ambiente, capaz de hacerlo saludable, satisfaciendo las necesidades humanas, el saneamiento es un servicio que se presta a un público, a un colectivo, independientemente de la naturaleza legal de su organización. El objetivo de este estudio es identificar el comportamiento de las principales variables que componen el elemento de saneamiento en una comunidad ribereña ubicada en un área ambientalmente protegida en la Amazonía brasileña. Este es un estudio longitudinal, descriptivo y ecológico, basado en la investigación de campo utilizando cuestionarios Revista Cerrados, Montes Claros/MG, v. 18, n. 1, p. 278-299, jan./jun.-2020. 
MARIOSA, D. F.; DE BENEDICTO, S. C.; GEORGES, M. R. R.; SUGAHARA, C. R.

A Agenda 2030 e o saneamento como indicador de sustentabilidade

estructurados y observación directa. Las condiciones de saneamiento encontradas fueron precarias o mínimamente garantizadas. El uso generalizado de "sumideros negros" para la eliminación de aguas residuales y la dificultad para suministrar agua potable eran preocupantes, lo que indica la necesidad de alguna forma de intervención que pueda corregir esta tendencia. Se concluye que el análisis de las condiciones de saneamiento de la comunidad, examinado en una escala de tiempo, permite la observación del comportamiento de las variables que componen el indicador utilizado y, por lo tanto, la detección de riesgos o vulnerabilidades que pueden, cuando persisten tendencias negativas encontrado, comprometer los aspectos económicos, sociales y ambientales que caracterizan la sostenibilidad de esas comunidades.

Palabras clave: Sostenibilidad. Saneamiento. Gestión de la salud. Unidades de Conservación. Comunidades ribereñas.

\section{INTRODUÇÃO}

O acesso ao fornecimento de água potável e às condições adequadas de saneamento faz parte da Agenda 2030 para o Desenvolvimento Sustentável, que prevê, em seu objetivo de número 6, "assegurar a disponibilidade e gestão sustentável da água e o saneamento para todos", orientando alcançar, até 2030, “o acesso universal e equitativo à água potável”, bem como "o acesso a saneamento e higiene adequados e equitativos para todos, e acabar com a defecação a céu aberto, com especial atenção para as necessidades das mulheres e meninas e daqueles em situação de vulnerabilidade" (NAÇÕES UNIDAS, 2015). O documento das Nações Unidas reforça que o padrão de qualidade de vida de uma população está diretamente relacionado à disponibilidade e qualidade da água consumida, dado que este é o recurso natural mais crítico à saúde humana, e mais suscetível a impor limites ao desenvolvimento (VICQ; LEITE, 2014).

A preocupação com a qualidade da água usada para o consumo humano e com o potencial deletério à saúde, movido pelos dejetos e resíduos orgânicos produzidos pelo homem, deriva, certamente, de uma questão ecológica, à medida que é provocada pela expansão demográfica da espécie humana (BOUABID; LOUIS, 2015). De fato, a importância para o bem-estar, conforto e qualidade de vida dos agregados humanos só se coloca quando os indivíduos passam a viver em comunidades ou unidades coletivas, passíveis de afrontar o ciclo de recuperação das fontes de abastecimento e de regeneração biológica (TRABUCCHI et al., 2012). De sorte que, enquanto o sistema ecológico mostra-se capaz de suportar o impacto das atividades humanas, estas podem seguir sem sobressaltos (SELBY; KAGAWA, Revista Cerrados, Montes Claros/MG, v. 18, n. 1, p. 278-299, jan./jun.-2020. 
MARIOSA, D. F.; DE BENEDICTO, S. C.; GEORGES, M. R. R.; SUGAHARA, C. R.

A Agenda 2030 e o saneamento como indicador de sustentabilidade

2013). Obviamente que os ciclos climáticos e geológicos continuam interferindo na estabilidade das sociedades; todavia, quando a maior parte da população mundial se concentra em cidades, outros perigos e ameaças despontam no horizonte da relação homem, sociedade e ambiente (MORACI; FAZIA, 2013).

O saneamento é visto como medida higienizadora do ambiente, capaz de torná-lo salubre para atender às necessidades humanas (SOUZA; FREITAS, 2009). Contudo, para o controle das doenças de veiculação hídrica, requer-se a interferência e regulação do Estado (SOARES; BERNARDES; CORDEIRO NETTO, 2002). A prestação de serviços de saneamento pelo Estado é um direito dos cidadãos brasileiros, previsto em texto constitucional que, entretanto, não alcança a totalidade de seus habitantes. A garantia legal do acesso não é, aqui por questões técnicas e financeiras, sinônimo de sua inteira fruição (SANTOS; MARQUES; DUARTE, 2011). Além da complexidade da estrutura física, necessária ao atendimento dos usuários, e dos recursos materiais, financeiros e humanos que, em geral, e em se tratando de fundos públicos, costumam ser escassos e heterogeneamente distribuídos (MEDEIROS et al., 2010), questões envolvendo a sustentabilidade ambiental, social e econômica precisam ser levadas em consideração em qualquer análise dessa ordem.

O Brasil ainda sofre com um agravante quadro de doenças relacionadas ao saneamento básico inadequado e precário, que tem como resultado o correspondente a, em média, 13.449 mortes por ano, ou seja, cerca de 1,31\% do total, sendo os estados amazônicos a região do país mais afetada (TEIXEIRA et al., 2014). A Amazônia possui componentes gigantescos se comparados aos demais biomas do planeta: ao lado da imensidão territorial, cita-se um patrimonio florestal considerável, rico em biodiversidade, e uma rede hidrográfica de dimensões continentais. Contrastando com outros espaços socioterritoriais brasileiros, os rios e igarapés são os caminhos e as estradas da população, e em suas margens estão seu sustento e os locais de encontro, de trocas e de atividade coletiva (FERREIRA; VENTICINQUE; ALMEIDA, 2005).

Para proteger a tênue estabilidade desses espaços territoriais e garantir que seus recursos possam ser racionalmente utilizados, o Poder Público identificou e delimitou algumas áreas geográficas, denominando-as Unidades de Conservação (UC's). Essas Unidades foram regulamentadas pela Lei do Sistema Nacional de Unidades de Conservação (SNUC), permitindo que, em alguns casos, os recursos naturais possam ser sustentavelmente utilizados, em particular pela população tradicional que, historicamente, ocupa essas terras, 
MARIOSA, D. F.; DE BENEDICTO, S. C.; GEORGES, M. R. R.; SUGAHARA, C. R.

A Agenda 2030 e o saneamento como indicador de sustentabilidade

como os ribeirinhos, indígenas, coletores, quilombolas, pescadores, entre outros (BRASIL, 2014).

O Estado do Amazonas, em particular, abriga, em seu interior, 111 Unidades de Conservação (UC's): 47 criadas por decretos federais, outras 41 por decretos estaduais e 23 por decretos municipais. Assim, cerca de 35\% do território estadual (mais de 44 milhões de hectares) é contemplado por áreas ambientalmente protegidas, das quais 18 são unidades de proteção integral e 58 de uso sustentável (BRASIL, 2014). Dentre estas, o presente estudo destaca a Reserva de Desenvolvimento Sustentável do Tupé - RDS do Tupé -, Unidade de Conservação de uso sustentável, localizada à margem esquerda do Rio Negro, distante cerca de $30 \mathrm{~km}$ do centro urbano de Manaus. Conforme estabelece o texto da Lei Federal $\mathrm{n}^{\circ}$ 9.985/2000 - SNUC (BRASIL, 2000) -, uma reserva de desenvolvimento sustentável é uma área natural que abriga populações tradicionais, cuja existência baseia-se em sistemas sustentáveis de exploração dos recursos naturais, desenvolvidos ao longo de gerações e adaptados às condições ecológicas locais, que desempenham um papel fundamental na proteção da natureza e na manutenção da diversidade biológica.

Ora, entre as comunidades ribeirinhas da Reserva de Desenvolvimento Sustentável do Tupé (SANTOS-SILVA; SCUDELLE; CAVALCANTI, 2011), as condições de saneamento encontradas, de imediato, apresentam-se como precárias, minimamente garantidas, e de difícil adequação normativa, dadas as características legais que regem as unidades de preservação ambiental (SILVA; REI, 2017).

Saneamento é um serviço que se presta a um público, a um coletivo, independente da natureza jurídica da organização. Diante disso, qualquer referência feita à qualidade do conjunto de serviços prestados na área de saneamento precisa, de um lado, abarcar os recursos materiais, humanos e as estruturas organizacionais disponibilizadas ao usuário; de outro, requer considerações sobre o universo sociocultural e territorial das relações entre o "clienteusuário" e a unidade prestadora de serviços (SPERLING; SPERLING, 2013). Tal condição exige, portanto, que a avaliação da qualidade seja construída a partir de uma métrica, referencial ou padrão que o pesquisador aplica às condições do cliente-usuário para apreender indiretamente esta dimensão.

A questão que se coloca neste artigo diz sobre a possibilidade de, a partir do uso os indicadores propostos pela Agenda 2030 para o Desenvolvimento Sustentável, avaliar a distância existente entre a realidade concreta de uma comunidade ribeirinha, integrante da 
MARIOSA, D. F.; DE BENEDICTO, S. C.; GEORGES, M. R. R.; SUGAHARA, C. R.

A Agenda 2030 e o saneamento como indicador de sustentabilidade

Reserva de Desenvolvimento Sustentável do Tupé, situada na área rural de Manaus, Amazonas, e os objetivos de desenvolvimento sustentável previstos para o item saneamento. Como ferramenta de gestão pública seria possível, então, com esta análise, sugerir medidas tópicas ou pontuais que pudessem minimizar suas prováveis consequências e potencial de risco à saúde humana, desde que dados básicos sobre o saneamento fossem disponibilizados aos agentes públicos, lideranças comunitárias e moradores.

À luz do que orienta a Agenda 2030 para o Desenvolvimento Sustentável, o objetivo geral do presente estudo é, portanto, identificar o comportamento das principais variáveis que compõem o item saneamento em uma comunidade ribeirinha localizada em área ambientalmente protegida na Amazônia brasileira. Em termos específicos, trata-se de examinar se as variáveis - de que trata o item saneamento - encontradas na Comunidade Ribeirinha do Nossa Senhora do Livramento (Livramento), em três momentos consecutivos, justificam a pertinência teórico-metodológica deste indicador de sustentabilidade socioambiental, e se é possível estimar o período necessário para a total efetivação das condições ótimas de saneamento nesta comunidade.

O presente artigo, além desta Introdução, apresenta, na sequência, a seção de Metodologia, em que descreve a área avaliada, o objeto de estudo, o plano amostral e as análises estatísticas correspondentes, além dos procedimentos para cálculo do saneamento. A seção de Apresentação e Análise dos Resultados descreve o que foi observado nas áreas avaliadas. Por fim, a seção de Conclusões, além de frisar o principal resultado obtido, apresenta as limitações do presente trabalho e a proposta para pesquisas futuras.

\section{MATERIAIS E MÉTODOS}

O presente texto apoia-se em estudo longitudinal, de natureza descritiva e ecológica (FACHIN, 2015), baseado em pesquisa de campo, com coleta de dados originais das moradias, prédios e construções, e observação direta das condições de saneamento da localidade escolhida. A área selecionada para estudo está localizada na Amazônia brasileira. Ladeando a margem esquerda do Rio Negro, a RDS do Tupé, conforme mostrado na Figura 1, é uma área de proteção ambiental inscrita na zona rural de Manaus (SANTOS-SILVA; SCUDELLE; CAVALCANTI, 2011). Seus 12.000 hectares de extensão, embora sejam territorialmente espaçosos, abrigam poucos moradores fixos no interior de seus limites. Algo 
MARIOSA, D. F.; DE BENEDICTO, S. C.; GEORGES, M. R. R.; SUGAHARA, C. R.

A Agenda 2030 e o saneamento como indicador de sustentabilidade

em torno de 1800 pessoas, distribuídas em seis comunidades, sendo cinco ribeirinhas (Tatulândia, São João do Tupé, Julião, Livramento e Agrovila) e uma de assentamento rural (Central). O presente estudo compreende apenas a comunidade ribeirinha do Livramento, população tradicional, assim chamada devido à influência do ritmo das águas em seu modo de vida, proporcionando, dessa forma, as condições para a construção de uma identidade coletiva semelhante ou com poucas variações em suas representações sociais (SERRA, 2002).

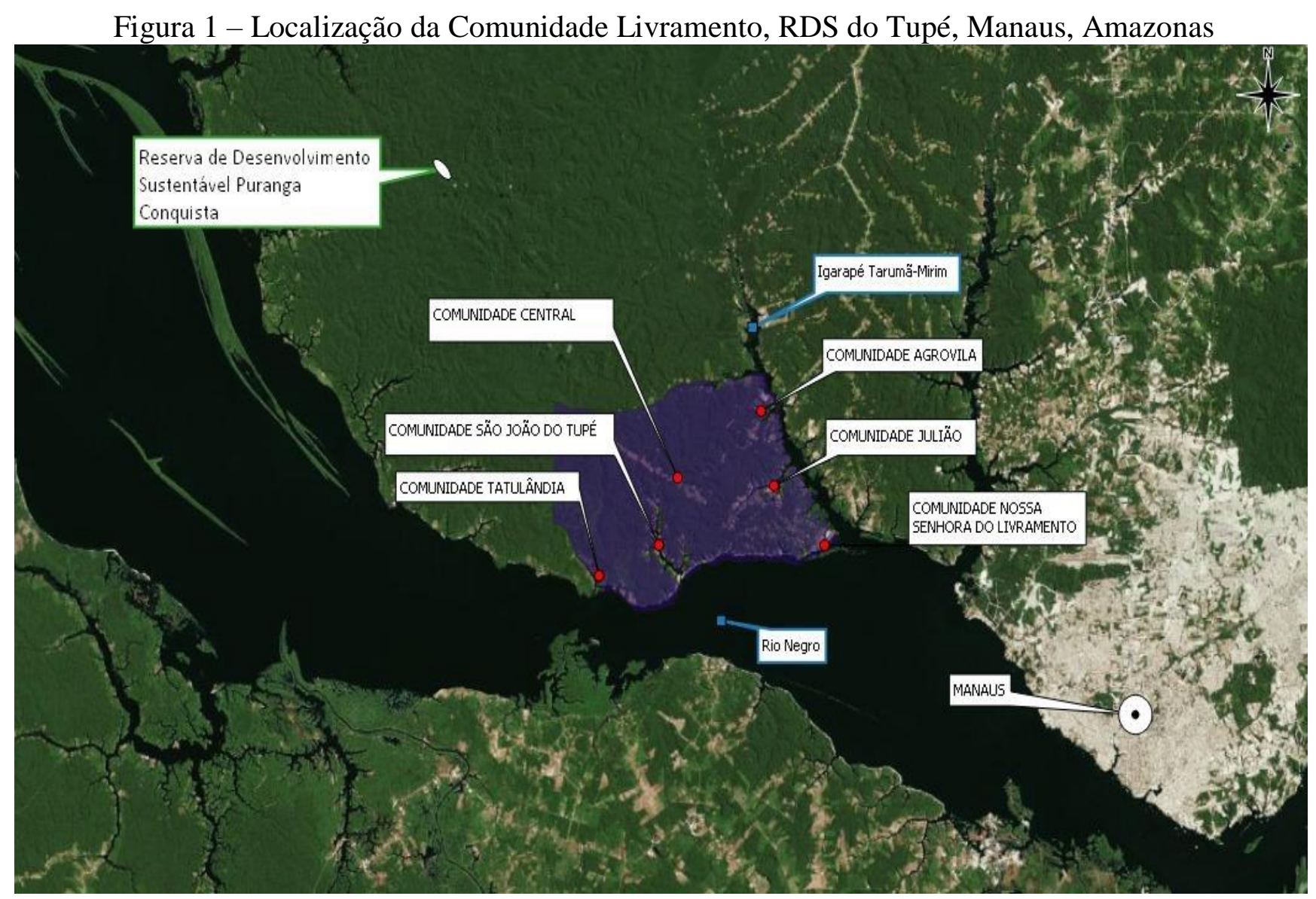

Fonte: Acervo Grupo de Pesquisa Biotupé (2018).

O plano amostral foi delineado a partir da distribuição espacial das construções localizadas na comunidade Livramento. Por se tratar de um número relativamente pequeno de unidades, concentradas na área central da comunidade, os dados foram coletados mediante o preenchimento de questionários estruturados, pretendendo-se alcançar a totalidade das construções. O levantamento dos dados foi realizado em três momentos distintos e subsequentes: de janeiro de 2010 a dezembro de 2010; de janeiro de 2012 a dezembro de 2013; e de janeiro a julho de 2016, no âmbito dos estudos de indicadores de qualidade 
MARIOSA, D. F.; DE BENEDICTO, S. C.; GEORGES, M. R. R.; SUGAHARA, C. R.

A Agenda 2030 e o saneamento como indicador de sustentabilidade

socioambiental (IQSA). (MARIOSA et al., 2014).

Para atender aos Objetivos de Desenvolvimento Sustentável, Agenda para 2030, em sua Meta 6 (Assegurar a disponibilidade e a gestão sustentável da água e saneamento para todos), são contemplados, para o caso estudado, os itens 6.1 (até 2030, alcançar o acesso universal e equitativo à água potável, segura e acessível para todos); 6.2 (até 2030, alcançar o acesso a saneamento e higiene adequados e equitativos para todos, e acabar com a defecação a céu aberto, com especial atenção para as necessidades das mulheres, meninas, e daqueles em situação de vulnerabilidade); e 6.3 (até 2030, melhorar a qualidade da água, reduzindo a poluição, eliminando despejo e minimizando a liberação de produtos químicos e materiais perigosos, reduzindo à metade a proporção de águas residuais não tratadas e aumentando substancialmente a reciclagem e reutilização segura, globalmente). Ademais, para o cálculo da variável "saneamento", considerou-se, neste estudo, o conjunto de procedimentos básicos que as comunidades ribeirinhas da RDS do Tupé costumam adotar em relação ao destino dado ao lixo doméstico, tratamento de esgoto, abastecimento de água potável, a existência e localização dos sanitários nas residências, da seguinte forma definida e ponderada:

- Destino do esgoto. A existência, nas moradias de forma adequada de tratamento do esgoto doméstico produzido, especificamente líquidos e excrementos humanos, pois essa ação contribui para impedir a proliferação de organismos patogênicos nos espaços da comunidade e em rios, lagos e nascentes. De acordo com o local em que são lançados os dejetos e resíduos sanitários (esgoto), tem-se um impacto maior ou menor nas fontes de contaminação. A situação ideal ocorre quando o esgoto produzido é destinado à rede pública e conduzido até uma estação de tratamento. Em áreas rurais isso não é possível, ou torna-se, muitas vezes, economicamente inviável, de modo que outras soluções costumam ser adotadas. Na RDS do Tupé tem-se os seguintes destinos: esgoto tratado (peso 10); fossa séptica ou cavidade no solo dotada de alguma forma de revestimento isolante (peso 8); fossa negra ou cavidade no solo sem qualquer forma de revestimento isolante (peso 4); rio (peso 2); ar livre (peso 0).

- Destino do lixo. Considera-se, neste item, o tratamento que é dado aos resíduos sólidos domésticos (lixo) acumulados nas residências e edificações; se há separação entre nãoorgânicos e orgânicos e sua posterior utilização em composteiras (peso 10); se é recolhido e enviado a aterro sanitário (8); se é queimado no local (6); quando é enterrado (4); ou 
MARIOSA, D. F.; DE BENEDICTO, S. C.; GEORGES, M. R. R.; SUGAHARA, C. R.

A Agenda 2030 e o saneamento como indicador de sustentabilidade

deixado a céu aberto (0).

- Água potável. Origem da água utilizada para consumo humano. Considerando-se o potencial impacto das doenças de veiculação hídrica, a água consumida pela população, sua origem e qualidade, são fatores essenciais para dimensionar a qualidade de vida que se quer observar. Na RDS do Tupé, dada a provável garantia de sua potabilidade em razão da distância de possíveis fontes de contaminação, temos a seguinte hierarquização quanto à origem do abastecimento de água potável: diretamente em poço artesiano comunitário (10); em reservatórios construídos para o armazenamento de água potável (8); retirada de poço particular (6); em rios e nascentes (4); outras formas de abastecimento (2).

- Sanitário. Diz respeito ao espaço, cômodo, construção ou ambiente destinado exclusivamente à higiene pessoal. Aqui, o critério de avaliação leva em conta sua existência e localização: se há mais de um (10); se está localizado no interior do imóvel (8); se em local separado da residência (6); se há formas improvisadas de fazer a higiene pessoal (2); ou se não possui espaço reservado para este fim (0).

- Vaso sanitário. Artefato especialmente desenhado para propiciar conforto ao indivíduo na hora de satisfazer suas necessidades fisiológicas de urinar e evacuar, evitando que animais domésticos, insetos e roedores tenham contato com os dejetos ali depositados, e se transformem em vetores de doenças e contaminações. Considera-se, na avaliação, a seguinte ordem classificatória: existência no imóvel de vaso cerâmico (10); caixa de madeira (8); ou a não existência de qualquer recipiente ou espécie de vaso sanitário (0).

Para a montagem das tabelas e construção dos indicadores de cada uma das variáveis, as notas dadas a cada quesito foram multiplicadas pela nota constante do Quadro 1, e ponderadas pelo número observado de residências na condição descrita. 
MARIOSA, D. F.; DE BENEDICTO, S. C.; GEORGES, M. R. R.; SUGAHARA, C. R.

A Agenda 2030 e o saneamento como indicador de sustentabilidade

Quadro 1 - Descrição, composição e peso atribuído às variáveis componentes do saneamento

\begin{tabular}{|c|c|c|}
\hline & & INFORMAÇÃO CONTIDA \\
\hline VARIAVEI & DESCRIÇÃO & $\begin{array}{l}\text { Peso relativo das res pos tas às variáveis } \\
\text { ótimo (10); bom (8); médio (6); regular (4); } \\
\text { péssimo (2); ou inexistente (0) }\end{array}$ \\
\hline \multirow{5}{*}{ Destino do esgoto } & \multirow{5}{*}{$\begin{array}{l}\text { Local onde são lançados os dejetos e } \\
\text { resíduos sanitários (esgoto) }\end{array}$} & esgoto tratado $(10)$ \\
\hline & & fossa séptica (8); \\
\hline & & fossa negra (4); \\
\hline & & rio (2); \\
\hline & & ar livre $(0)$ \\
\hline \multirow{5}{*}{ Destino do lixo } & \multirow{5}{*}{$\begin{array}{l}\text { Tratamento que é dado aos resíduos sólidos } \\
\text { domésticos (lixo) acumulados nas habitações }\end{array}$} & composteira $(10)$ \\
\hline & & recolhido (8); \\
\hline & & queimado $(6)$; \\
\hline & & enterrado (4) \\
\hline & & céu aberto $(0)$. \\
\hline \multirow{5}{*}{ Água potável } & \multirow{5}{*}{$\begin{array}{l}\text { Fonte ou origem do abastecimento de água } \\
\text { potável }\end{array}$} & poço artes iano comunitário (10); \\
\hline & & reservatório (8); \\
\hline & & poço particular (6); \\
\hline & & rio/nas cente (4); \\
\hline & & outro (2). \\
\hline \multirow{5}{*}{ Sanitário } & \multirow{5}{*}{$\begin{array}{l}\text { Construção ou ambiente destinado } \\
\text { exclusivamente à higiene pessoal }\end{array}$} & mais de um (10); \\
\hline & & interior do imóvel (8); \\
\hline & & separado da residência (6); \\
\hline & & formas improvisadas (2); \\
\hline & & não possui $(0)$. \\
\hline \multirow{3}{*}{ Vaso sanitário } & \multirow{3}{*}{$\begin{array}{c}\text { Artefato especialmente desenhado para } \\
\text { propiciar conforto ao individuo na hora de } \\
\text { satis fazer suas necessidades fisiológicas de } \\
\text { urinar e evacuar }\end{array}$} & vaso cerâmico (10); \\
\hline & & caixa de madeira (8); \\
\hline & & não possui (0). \\
\hline
\end{tabular}

Fonte: Elaborado pelos autores, a partir da Meta 6 da Agenda 2030 para o Desenvolvimento Sustentável (NAÇÕES UNIDAS, 2015).

\section{RESULTADOS E DISCUSSÃO}

Na Comunidade Ribeirinha do Livramento, RDS do Tupé, Manaus, Amazonas, no período de janeiro a julho de 2010, 124 edificações foram observadas. Repetindo-se o mesmo procedimento, no período de janeiro a dezembro de 2013, foram coletados dados em 192 edificações da mesma comunidade. E, por fim, no período de janeiro a dezembro de 2016, foram coletados dados em 210 edificações.

Para cada uma das cinco variáveis foi calculado o indicador estratificado por ano, a começar pela variável Destino do Esgoto. Depois, foi feita a análise conjunta da dimensão "saneamento", à luz dos Objetivos do Desenvolvimento Sustentável e sua factibilidade frente às metas definidas para o ano de 2030.

Os dados coletados na variável Destino do Esgoto foram sistematizados na Tabela 1, cabendo algumas observações acerca dessa variável. A mais importante, talvez, seja Revista Cerrados, Montes Claros/MG, v. 18, n. 1, p. 278-299, jan./jun.-2020. 
MARIOSA, D. F.; DE BENEDICTO, S. C.; GEORGES, M. R. R.; SUGAHARA, C. R.

A Agenda 2030 e o saneamento como indicador de sustentabilidade

o fato de não se ter encontrado edificações com esgoto tratado nos três períodos pesquisados. Já em relação ao uso de fossa séptica, não foram encontradas edificações que a utilizam nos anos de 2010 e 2013, mas foram observadas 13 edificações que a adotam em 2016. Com relação ao uso de fossa negra, a pesquisa encontrou 75 edificações que a usam em 2010, 136 edificações em 2013 e 82 edificações em 2016. Com relação ao despejo do esgoto no rio, a pesquisa encontrou 2 edificações nesta condição em 2010, 5 edificações em 2013 e somente uma edificação que despeja o esgoto no rio no ano de 2016. Por fim, foram encontradas 6 edificações que despejam o esgoto ao ar livre no ano de 2010, 9 edificações em 2013 e 8 edificações nesta condição na pesquisa de 2016.

Tabela 1 - Resultados Destino do Esgoto

\begin{tabular}{|c|c|c|c|c|c|c|c|}
\hline \multirow{2}{*}{ DESTINO DO ESGOTO } & \multirow{2}{*}{$\begin{array}{c}\text { Peso } \\
\text { Aplicado }\end{array}$} & \multicolumn{2}{|c|}{2010} & \multicolumn{2}{|c|}{2013} & \multicolumn{2}{|c|}{2016} \\
\hline & & Casos & Total & Casos & Total & Casos & Total \\
\hline Tratado & 10 & 0 & 0 & 0 & 0 & 0 & 0 \\
\hline Fossa Séptica & 8 & 0 & 0 & 0 & 0 & 13 & 104 \\
\hline Fossa Negra & 4 & 75 & 300 & 136 & 544 & 82 & 328 \\
\hline Rio & 2 & 2 & 4 & 5 & 10 & 1 & 2 \\
\hline Ar Livre & 0 & 6 & 0 & 9 & 0 & 8 & 0 \\
\hline \multicolumn{2}{|l|}{ SUB TOTAL } & 83 & 304 & 150 & 554 & 104 & 434 \\
\hline \multicolumn{2}{|c|}{$I Q S A=\frac{\sum P \text { Peso } \cdot \text { Caso }}{10 \cdot \text { Total Casos }}$} & \multicolumn{2}{|c|}{0,3663} & \multicolumn{2}{|c|}{0,3693} & \multicolumn{2}{|c|}{0,4173} \\
\hline \multicolumn{2}{|l|}{ Não Informado } & \multicolumn{2}{|c|}{41} & \multicolumn{2}{|c|}{42} & \multicolumn{2}{|c|}{106} \\
\hline \multicolumn{2}{|c|}{ TOTAL MORADIAS PESQUISADAS } & \multicolumn{2}{|c|}{124} & \multicolumn{2}{|c|}{192} & \multicolumn{2}{|c|}{210} \\
\hline
\end{tabular}

Fonte: Elaborada pelos autores.

A partir da quantidade de edificações observadas em cada categoria, foi calculado o indicador de qualidade socioambiental (IQSA) para a variável Destino do Esgoto, multiplicando a quantidade observada pelo respectivo peso atribuído, e dividindo pela totalidade dos casos, considerando a melhor situação possível. Assim, obteve-se, para os casos efetivamente observados, o valor de 0,3663 para o IQSA na pesquisa 2010, o valor 0,3693 para o ano de 2013 e o valor de 0,4173 para 2016.

Lamenta-se o fato de a pesquisa ter encontrado edificações que declaradamente lançam o esgoto doméstico ao "ar livre" nos três anos (Tabela 1). Essa condição precária em relação ao destino do esgoto se acentua ainda mais ao se incluir na análise os dados da prática mais comum adotada, que é a utilização da "fossa negra" como destinação final dos dejetos 
MARIOSA, D. F.; DE BENEDICTO, S. C.; GEORGES, M. R. R.; SUGAHARA, C. R.

A Agenda 2030 e o saneamento como indicador de sustentabilidade

humanos. "Fossa negra" nada mais é que um buraco ou fossa cavada no chão, sobre o qual os moradores colocam uma tampa vazada, assentam um vaso cerâmico ou caixa de madeira, para que seus ocupantes possam evacuar sentados. Aliás, durante o período considerado, o número de sanitários que adotaram a caixa de madeira como recurso de conforto subiu de 1 para 14, e depois caiu para 9, em 2016. Quando a "fossa negra" enche, abre-se uma nova em um local próximo da casa, por isso o grande número de sanitários localizados fora do imóvel: 47, 52 e 43 nos anos de 2010, 2013 e 2016, respectivamente.

Por não receber nenhum tipo de tratamento, a prática de se abrir "fossas negras", ou mesmo com algum rudimento de isolamento nas "fossas sépticas", pode, potencialmente, comprometer lençóis freáticos e cursos d'água próximos, e também servir de criadouro para roedores e animais peçonhentos. Os casos de esgoto tratado permaneceram com nenhuma observação nos três períodos pesquisados.

De forma análoga, a Tabela 2 apresenta os dados coletados para a variável Destino do Lixo e o subsequente cálculo do IQSA. Observa-se um aumento do IQSA de 2013, de 0,7635, em relação a 2010, de 0,6987; depois, tem-se uma queda em 2016, para 0,6936, próximo aos patamares de 2010.

Tabela 2 - Resultados Destino do Lixo

\begin{tabular}{|c|c|c|c|c|c|c|c|}
\hline \multirow{2}{*}{ DESTINO DO LIXO } & \multirow{2}{*}{ Peso Aplicado } & \multicolumn{2}{|c|}{2010} & \multicolumn{2}{|c|}{2013} & \multicolumn{2}{|c|}{2016} \\
\hline & & Casos & Total & Casos & Total & Casos & Total \\
\hline Composteira & 10 & 0 & 0 & 0 & 0 & 4 & 40 \\
\hline Recolhido & 8 & 57 & 456 & 148 & 1184 & 64 & 512 \\
\hline Queimado & 4 & 17 & 68 & 7 & 28 & 24 & 96 \\
\hline Enterrado & 2 & 0 & 0 & 1 & 2 & 2 & 4 \\
\hline Ceu Aberto & 0 & 1 & 0 & 3 & 0 & 0 & 0 \\
\hline \multicolumn{2}{|c|}{ SUB TOTAL } & 75 & 524 & 159 & 1214 & 94 & 652 \\
\hline \multicolumn{2}{|c|}{$I Q S A=\frac{\sum P \text { Peso } \cdot \text { Caso }}{10 \cdot \text { Total Casos }}$} & \multicolumn{2}{|c|}{0,6987} & \multicolumn{2}{|c|}{0,7635} & \multicolumn{2}{|c|}{0,6936} \\
\hline \multicolumn{2}{|c|}{ Não Informado } & \multicolumn{2}{|c|}{49} & \multicolumn{2}{|c|}{33} & \multicolumn{2}{|c|}{116} \\
\hline \multicolumn{2}{|c|}{ TOTAL MORADIAS PESQUISADAS } & \multicolumn{2}{|c|}{124} & \multicolumn{2}{|c|}{192} & \multicolumn{2}{|c|}{210} \\
\hline
\end{tabular}

Fonte: Elaborada pelos autores.

O destino dado ao lixo doméstico, ou seja, aos resíduos produzidos em cada uma das casas, passou por alguma melhoria, como a observação de 4 composteiras em 2016, frente 
a nenhuma observação nos anos anteriores, bem como o fim do despejo a céu aberto, observado em 2010, e não mais nos anos seguintes. Pelo fato de estar localizada em área ambientalmente protegida, verifica-se, por parte dos órgãos de vigilância ambiental, visitantes e moradores, uma preocupação constante com o descarte adequado do lixo produzido na comunidade. O indicador de qualidade socioambiental (IQSA) para a variável Água Potável obteve um resultado de 0,547 para o ano de 2010; 0,6592 para o ano de 2013, e o valor de 0,6376 para 2016. A Tabela 3 apresenta tais resultados.

Tabela 3 - Resultados Água Potável

\begin{tabular}{|c|c|c|c|c|c|c|c|}
\hline \multirow{2}{*}{ ÁGUA POTÁVEL } & \multirow{2}{*}{$\begin{array}{c}\text { Peso } \\
\text { Aplicado }\end{array}$} & \multicolumn{2}{|c|}{2010} & \multicolumn{2}{|c|}{2013} & \multicolumn{2}{|c|}{2016} \\
\hline & & Casos & Total & Casos & Total & Casos & Total \\
\hline Poço Comunitario & 10 & 3 & 30 & 19 & 190 & 14 & 140 \\
\hline Reservatorio & 8 & 3 & 24 & 23 & 184 & 14 & 112 \\
\hline Poço Particular & 6 & 58 & 348 & 98 & 588 & 69 & 414 \\
\hline Rio/Nascente & 4 & 7 & 28 & 8 & 32 & 20 & 80 \\
\hline Outro & 2 & 12 & 24 & 4 & 8 & 0 & 0 \\
\hline \multicolumn{2}{|c|}{ SUB TOTAL } & 83 & 454 & 152 & 1002 & 117 & 746 \\
\hline \multicolumn{2}{|c|}{$\frac{\sum \text { Peso } \cdot \text { Caso }}{10 \cdot \text { Total Casos }}$} & \multicolumn{2}{|c|}{0,5470} & \multicolumn{2}{|c|}{0,6592} & \multicolumn{2}{|c|}{0,6376} \\
\hline \multicolumn{2}{|c|}{ Não Informado } & \multicolumn{2}{|c|}{41} & \multicolumn{2}{|c|}{40} & \multicolumn{2}{|c|}{93} \\
\hline \multicolumn{2}{|c|}{ TOTAL MORADIAS PESQUISADAS } & \multicolumn{2}{|c|}{124} & \multicolumn{2}{|c|}{192} & \multicolumn{2}{|c|}{210} \\
\hline
\end{tabular}

Fonte: Elaborada pelos autores.

Água potável é um bem de insofismável necessidade humana. Não há condições de sobrevivência sem que uma quantidade adequada de água tratada ou com um mínimo de potabilidade esteja à disposição da comunidade. Ora, embora situados à margem de rios e igarapés com grandes caudais e volumosos recursos hídricos, não há como garantir que a água consumida na Comunidade Ribeirinha do Livramento esteja própria para o consumo humano. As fontes em que os moradores a obtém são majoritariamente poços particulares ou comunitários, ou seja, retirados dos lençóis freáticos do subsolo.

Já o indicador de qualidade socioambiental (IQSA) para a variável Sanitário obteve os resultados de 0,5800 para o ano de 2010, o valor 0,5924 para o ano de 2013, e o valor de 0,7237 para o ano de 2016, conforme se observa na Tabela 4. 
MARIOSA, D. F.; DE BENEDICTO, S. C.; GEORGES, M. R. R.; SUGAHARA, C. R.

A Agenda 2030 e o saneamento como indicador de sustentabilidade

Tabela 4 - Resultados Sanitário

\begin{tabular}{|c|c|c|c|c|c|c|c|}
\hline \multirow{2}{*}{ SANITÁRIO } & \multirow{2}{*}{ Peso Aplicado } & \multicolumn{2}{|c|}{2010} & \multicolumn{2}{|c|}{2013} & \multicolumn{2}{|c|}{2016} \\
\hline & & Casos & Total & Casos & Total & Casos & Total \\
\hline Mais de 1 & 10 & 1 & 10 & 4 & 40 & 12 & 120 \\
\hline No Imóvel & 8 & 36 & 288 & 73 & 584 & 77 & 616 \\
\hline Separado & 6 & 47 & 282 & 52 & 312 & 43 & 258 \\
\hline Outro & 2 & 0 & 0 & 0 & 0 & 6 & 12 \\
\hline Não Possui & 0 & 16 & 0 & 29 & 0 & 1 & 0 \\
\hline \multicolumn{2}{|c|}{ SUB TOTAL } & 100 & 580 & 158 & 936 & 139 & 1006 \\
\hline \multicolumn{2}{|c|}{$I Q S A=\frac{\sum P e s o \cdot \operatorname{Caso}}{10 \cdot \text { Total Casos }}$} & \multicolumn{2}{|c|}{0,5800} & \multicolumn{2}{|c|}{0,5924} & \multicolumn{2}{|c|}{0,7237} \\
\hline \multicolumn{2}{|c|}{ Não Informado } & \multicolumn{2}{|c|}{24} & \multicolumn{2}{|c|}{34} & \multicolumn{2}{|c|}{71} \\
\hline \multicolumn{2}{|c|}{ TOTAL MORADIAS PESQUISADAS } & \multicolumn{2}{|c|}{124} & \multicolumn{2}{|c|}{192} & \multicolumn{2}{|c|}{210} \\
\hline
\end{tabular}

Fonte: Elaborada pelos autores.

A importância do descarte adequado dos dejetos humanos passa, certamente, pela construção de espaços higienicamente apropriados para este fim. No todo, percebe-se uma melhoria dessas condições nos imóveis residenciais localizados na Comunidade Ribeirinha do Livramento; entretanto, como observado na Tabela 1, o destino do esgoto que a deveria complementar não oferece as condições para seu devido tratamento antes de retornar ao ambiente. Por fim, para o indicador de qualidade socioambiental (IQSA) para a variável Vaso Sanitário, apresentado na Tabela 5, obteve-se os resultados de 0,6045 para o ano de 2010, o valor 0,6949 para o ano de 2013, e o valor de 0,8757 para o ano de 2016.

Tabela 5 - Resultados Vaso Sanitário

\begin{tabular}{|c|c|c|c|c|c|c|c|}
\hline \multirow{2}{*}{ VASO SANITARIO } & \multirow{2}{*}{$\begin{array}{c}\text { Peso } \\
\text { Aplicado }\end{array}$} & \multicolumn{2}{|c|}{2010} & \multicolumn{2}{|c|}{2013} & \multicolumn{2}{|c|}{2016} \\
\hline & & Casos & Total & Casos & Total & Casos & Total \\
\hline Vaso Cerâmico & 10 & 53 & 530 & 84 & 840 & 83 & 830 \\
\hline Caixa de Madeira & 8 & 1 & 8 & 14 & 112 & 9 & 72 \\
\hline Não Possui & 0 & 35 & 0 & 39 & 0 & 11 & 0 \\
\hline \multicolumn{2}{|c|}{ SUB TOTAL } & 89 & 538 & 137 & 952 & 103 & 902 \\
\hline \multicolumn{2}{|c|}{$I Q S A=\frac{\sum P \text { Peso } \cdot \text { Caso }}{10 \cdot \text { Total Casos }}$} & \multicolumn{2}{|c|}{0,6045} & \multicolumn{2}{|c|}{0,6949} & \multicolumn{2}{|c|}{0,8757} \\
\hline \multicolumn{2}{|c|}{ Não Informado } & \multicolumn{2}{|c|}{35} & \multicolumn{2}{|c|}{55} & \multicolumn{2}{|c|}{107} \\
\hline \multicolumn{2}{|c|}{ TOTAL MORADIAS PESQUISADAS } & \multicolumn{2}{|c|}{124} & \multicolumn{2}{|c|}{192} & \multicolumn{2}{|c|}{210} \\
\hline
\end{tabular}

Fonte: Elaborada pelos autores. 
MARIOSA, D. F.; DE BENEDICTO, S. C.; GEORGES, M. R. R.; SUGAHARA, C. R.

A Agenda 2030 e o saneamento como indicador de sustentabilidade

Nota-se, na Tabela 5, que o uso de vaso cerâmico, em ambientes construídos para tal fim (Tabela 4), está bem disseminado na Comunidade Ribeirinha do Livramento, embora o número de casos "não informado" possa trazer alguma insegurança a esta colocação. Ressaltese, entretanto, que são muitas as casas que encontram-se abandonadas por seus moradores, ou que são ocupadas de forma sazonal e esporádica, devido aos seus ocupantes manterem residência fixa em Manaus (MARIOSA et al., 2014).

Em síntese, a Figura 2 ilustra os valores do IQSA obtidos para cada uma das cinco variáveis em cada um dos três períodos em que a pesquisa foi realizada. Observa-se que as variáveis Vaso Sanitário, Sanitário e Destino do Esgoto evoluíram ao longo dos anos, enquanto Destino do Lixo e Água Potável não.

Figura 2 - Gráfico comparativo do IQSA por variável por ano

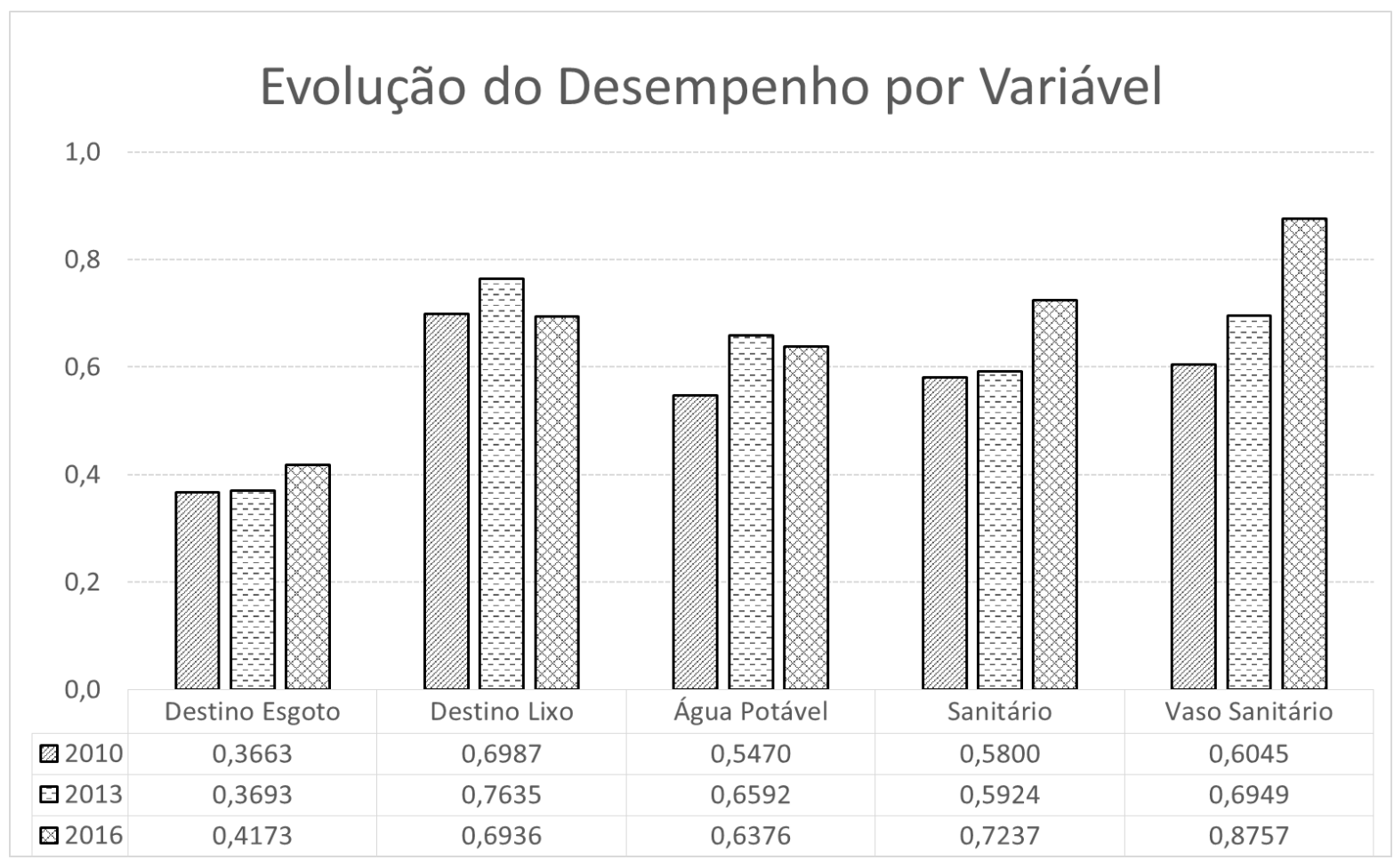

Fonte: Elaborada pelos autores.

Para que a análise até aqui empreendida se complete, é necessário, porém, acrescentar os elementos observados nas comunidades e compará-los com a Meta 6 dos Objetivos de Desenvolvimento Sustentável da Agenda 2030. 
MARIOSA, D. F.; DE BENEDICTO, S. C.; GEORGES, M. R. R.; SUGAHARA, C. R.

A Agenda 2030 e o saneamento como indicador de sustentabilidade

\section{Projeção Futura para o Objetivo de Desenvolvimento Sustentável 6 da Agenda 2030}

Diante do valor IQSA para cada variável, a partir da média destes pelo número de edificações respondentes, calcula-se o IQSA total da Dimensão Moradia. Este cálculo da média ponderada do IQSA da Dimensão Moradia está apresentado na Tabela 6, em que se observa claramente um aumento deste indicador ao longo dos anos em que a pesquisa foi feita. Em 2010, o IQSA geral da Dimensão Moradia foi de 0,5581, já no ano de 2013 o IQSA subiu para 0,6161; e em 2016 foi para 0,6715 .

Tabela 6 - Cálculo do IQSA da Dimensão Moradia

\begin{tabular}{c|r|r|r|r|r|r}
\hline \multirow{2}{*}{ DIMENSÃO MORADIA } & \multicolumn{2}{|c|}{2010} & \multicolumn{2}{|c|}{2013} & \multicolumn{2}{|c}{2016} \\
\cline { 2 - 7 } & IQSA & Casos & IQSA & Casos & IQSA & Casos \\
\hline Destino do Esgoto & 0,3663 & 83 & 0,3693 & 150 & 0,4173 & 104 \\
\hline Destino do Lixo & 0,6987 & 75 & 0,7635 & 159 & 0,6936 & 94 \\
\hline Água Potável & 0,5470 & 83 & 0,6592 & 152 & 0,6376 & 117 \\
\hline Sanitário & 0,5800 & 100 & 0,5924 & 158 & 0,7237 & 139 \\
\hline Vaso Sanitário & 0,6045 & 89 & 0,6949 & 137 & 0,8757 & 103 \\
\hline IQSA MORADIA & \multicolumn{2}{|c|}{$\mathbf{0 , 5 5 8 1}$} & \multicolumn{2}{|c}{$\mathbf{0 , 6 1 6 1}$} & \multicolumn{2}{|c}{$\mathbf{0 , 6 7 1 5}$} \\
\hline
\end{tabular}

Fonte: Elaborada pelos autores.

A partir dos valores do IQSA para cada um dos três períodos, procedeu-se uma projeção linear a partir de um modelo de regressão linear simples, para verificar se este indicador atingiria o valor pleno até 2030. A Figura 3 exibe os valores do IQSA obtidos para os três anos, e a linha de tendência, obtida com o auxílio de planilha eletrônica.

Utilizando-se a equação obtida pela rgressão linear aimples, observa-se que, se este padrão persistir, no ano de 2030 o indicador IQSA da Dimensão Moradia estará acima de 0,9, atingindo 0,965. Também utilizando a equação da regressão, calcula-se que o IQSA da Dimensão Moradia atingirá o valor 1 no final do ano de 2031, persistindo tal padrão. 
MARIOSA, D. F.; DE BENEDICTO, S. C.; GEORGES, M. R. R.; SUGAHARA, C. R.

A Agenda 2030 e o saneamento como indicador de sustentabilidade

Figura 3 - Projeção Linear Futura do IQSA

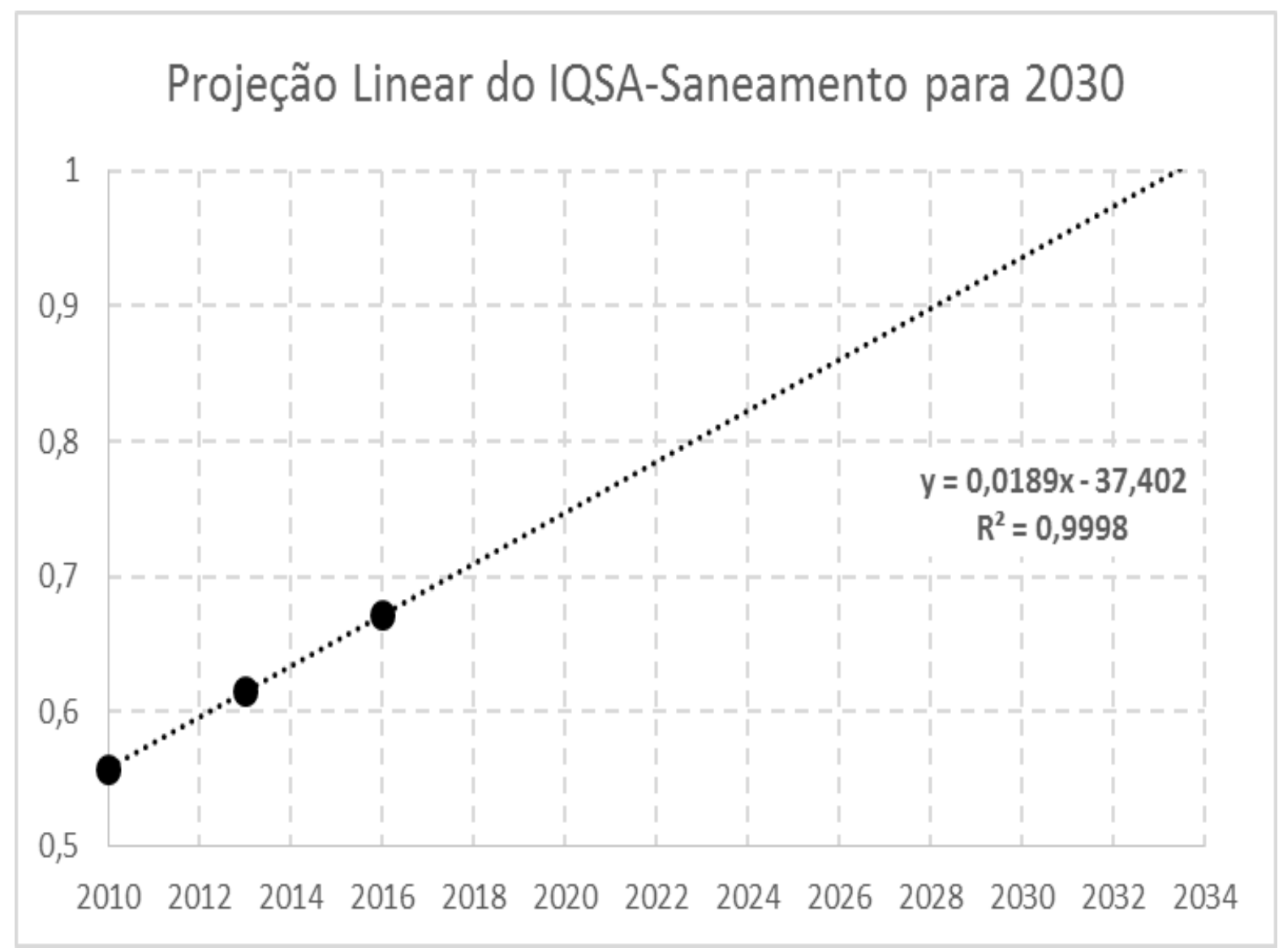

Fonte: Elaborada pelos autores.

A estatística da regressão ao quadrado de 0,9998 é excelente! Quase um! Seria uma regressão perfeita, em que os três pontos pertenceriam à mesma reta. No entanto, é preciso relevar a amostra de apenas três períodos, o que não permite precisão na projeção, porém já revela informações significativas, como se observa na Figura 4.

Utilizando o mesmo artifício da regressão linear simples e reconhecendo suas limitações quando a amostra é diminuta, a Figura 4 projeta a tendência, para o ano de 2030, do IQSA para cada uma das cinco variáveis que compõem a Dimensão Moradia, a partir dos resultados obtidos nas pesquisas realizadas em 2010, 2013 e 2016. 
MARIOSA, D. F.; DE BENEDICTO, S. C.; GEORGES, M. R. R.; SUGAHARA, C. R.

A Agenda 2030 e o saneamento como indicador de sustentabilidade

Figura 4 - Projeção Linear Futura do IQSA por Variável

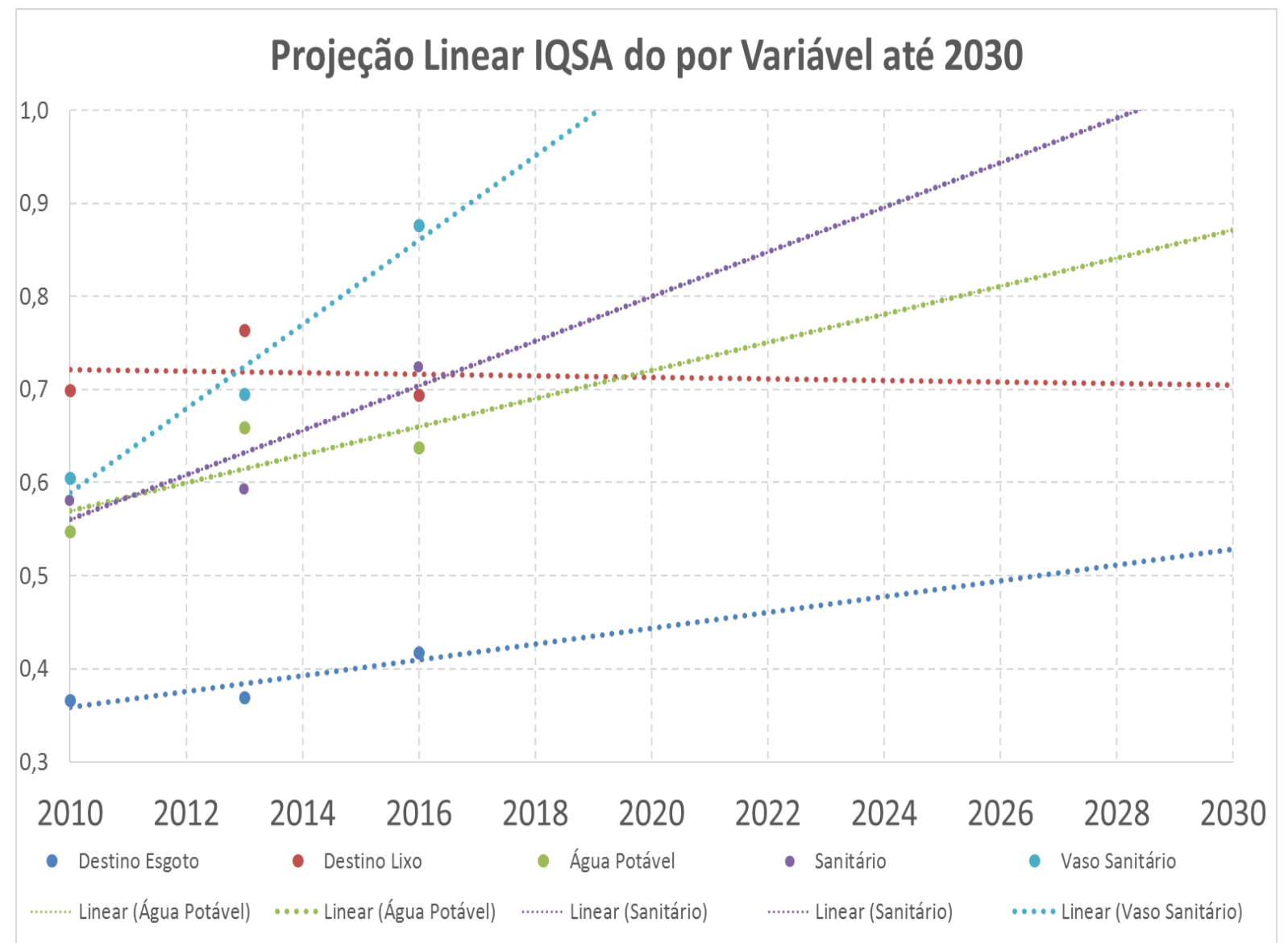

Fonte: Elaborada pelos autores.

Neste gráfico, pode-se observar que a variável Vaso Sanitário é a primeira a atingir a meta de 1, por volta do ano de 2019. A variável Sanitário também atingirá a meta de 1 antes de 2030, porém mais próximo, em 2028. Já a variável Água Potável não atingirá a meta de 1 em 2030, estando próximo de 0,85, assim como a variável Destino de Esgoto que, em 2030, estará pouco acima de 0,5. Por fim, a variável Destino de Lixo estará pior em 2030 do que se encontra atualmente, se a tendência persistir.

\section{CONCLUSÕES}

$\mathrm{Na}$ análise dos aspectos gerais das condições de saneamento da população da Comunidade Ribeirinha do Livramento, RDS do Tupé, Manaus/AM, o fato dos resultados apontarem uma razoável melhora nos indicadores talvez não seja suficiente para as mudanças no repertório de políticas públicas, especialmente de suporte, prevenção e assistência à saúde. 
MARIOSA, D. F.; DE BENEDICTO, S. C.; GEORGES, M. R. R.; SUGAHARA, C. R.

A Agenda 2030 e o saneamento como indicador de sustentabilidade

Investimentos em saneamento básico podem ajudar a reduzir índices de ocorrência de doenças de veiculação hídrica, ainda verificados na região. Entretanto, o clima tropical da Amazônia não é favorável à estabilização dessas condições. Com altos índices pluviométricos e temperaturas elevadas durante todo o ano, as localidades necessitam de medidas específicas e pontuais, que vão além dos tradicionais mecanismos de saneamento e controle epidemiológico aplicados em outras áreas de habitação, exclusivamente periféricas, rurais ou florestadas. Exige-se, antes, a compreensão das variáveis operativas no território e, ainda que garantida sua observância pelo texto constitucional, a disposição política para agir.

Como principal resultado deste estudo tem-se que a análise das condições de saneamento da Comunidade Livramento, examinadas em escala temporal, permite a observação do comportamento das variáveis que compõem o indicador utilizado e, dessa forma, a detecção de riscos ou de vulnerabilidades que podem, se persistirem as tendências negativas encontradas, comprometer aspectos econômicos, sociais e ambientais que caracterizam a sustentabilidade daquelas comunidades. De outra forma, entre os principais benefícios que se pode obter do presente trabalho está a possibilidade de utilizá-lo para uma gestão mais efetiva dos recursos alocados em saneamento e, acessoriamente, dos gastos em saúde, especialmente com o tratamento de doenças de veiculação hídrica, ou no controle de vetores de doenças infectocontagiosas.

As principais limitações do estudo se relacionam ao fato de serem utilizadas para análise apenas as condições de saneamento em uma área de características rurais, parcamente povoada e ambientalmente resiliente ao impacto das atividades antrópicas. Assim, como proposta para pesquisas futuras, sugere-se a continuidade dos estudos na região, especialmente relacionados à ocorrência de doenças evitáveis através da melhoria das condições de saneamento geral das comunidades e, ainda, a repetição da mesma metodologia em ambientes semelhantes.

\section{REFERÊNCIAS}

BOUABID, Ali; LOUIS, Garrick. Capacity factor analysis for evaluating water and sanitation infrastructure choises for developing communities. Journal of Environmental

Management, v.161, p. 335-343, 2015.

BRASIL. MINISTÉRIO DO MEIO AMBIENTE. Lei N 9.985, de 18 de julho de 2000. Sistema Nacional de Unidade de Conservação da Natureza - SNUC. Brasilia - DF: MMA, 2000 . 
MARIOSA, D. F.; DE BENEDICTO, S. C.; GEORGES, M. R. R.; SUGAHARA, C. R. A Agenda 2030 e o saneamento como indicador de sustentabilidade

BRASIL. MINISTÉRIO DO MEIO AMBIENTE. Sistema Nacional de Unidades de Conservação da Natureza. Brasília: MMA/SBF, 2011. 80 p.

BRASIL. MINISTÉRIO DO MEIO AMBIENTE. Cadastro nacional de unidades de conservação. 2014. Disponível em: <http://www.mma.gov.br/areas-protegidas/cadastronacional-de-ucs>. Acesso em: 23 jul. 2018.

FACHIN, Odília. Fundamentos de metodologia. 5. ed. São Paulo: Saraiva, 2015. 210 p.

FERREIRA, Leandro Valle; VENTICINQUE, Eduardo; ALMEIDA, Samuel. et al. O desmatamento na Amazônia e a importância das áreas protegidas. Estudos Avançados, v. 19, n. 53, p. 157-166, 2005.

MARIOSA, Duarcides Ferreira; SILVA, Edinaldo Nelson dos Santos; GASPARINI, Leandro; REIS Jr., Alfredo Morel. Ribeirinhos do Rio Negro: um estudo da qualidade socioambiental. Curitiba: Editora CRV, 2014. 158 p.

MEDEIROS, Flávia A.; ARAÚJO-SOUZA, Georgia C.; ALBUQUERQUE-BARBOSA, Aldenísia A.; CLARA-COSTA, Iris do Céu. Acolhimento em uma Unidade Básica de Saúde: a satisfação do usuário em foco. Revista de Salud Pública, v. 12, n. 3, p. 402-13, 2010.

MORACI, Francesca; FAZIA, Celestina. The smart cities and the challengers sustainability. TeMA - Journal of Land Use, Mobility and Environment, v. 6, n. 1, p. 35-45, 2013.

NAÇÕES UNIDAS. Transformando nosso mundo: a agenda 2030 para o desenvolvimento sustentável. 2015. Disponível em: <http://nacoesunidas.org/pos2015/agenda2030/>. Acesso em: 13 set. 2016.

SANTOS, Fernanda Barbosa; MARQUES, Leonardo Augusto Marinho; DUARTE, Hugo Garcez. Direitos Fundamentais: a busca por sua efetivação. Âmbito Jurídico, v.14, n. 92, p. $1-13,2011$.

SANTOS-SILVA, Edinaldo Nelson; SCUDELLE, Veridiana Vizoni; CAVALCANTI, Mauro José (Eds.). BioTupé: meio físico, diversidade biológica e sociocultural do baixo Rio Negro, Amazônia Central. Manaus: Rizoma Editorial, 2011. v. 3.

SERRA, N. E. M. No ritmo das águas. Presença Revista de Educação, Cultura e Meio Ambiente, v. 4, n. 24, p. 1-13, 2002.

SELBY, David; KAGAWA, Fumiyo. Mudança climática em sala de aula. Brasília: UNESCO, 2014. 374 p.

SILVA, Antonio Carlos Nisoli Pereira; REI, Fernando Cardozo Fernandes. O Direito Ambiental Internacional: um olhar da Ciência Pós-Normal. Revista Eletrônica Direito e Sociedade - REDES, v. 5, n. 2, p. 11-22, 2017.

SOARES, Sérgio R. A.; BERNARDES, Ricardo S.; CORDEIRO NETO, Oscar M. Relações entre saneamento, saúde pública e meio ambiente: elementos para formulação de um modelo Revista Cerrados, Montes Claros/MG, v. 18, n. 1, p. 278-299, jan./jun.-2020. 
MARIOSA, D. F.; DE BENEDICTO, S. C.; GEORGES, M. R. R.; SUGAHARA, C. R.

A Agenda 2030 e o saneamento como indicador de sustentabilidade

de planejamento em saneamento. Cadernos de Saúde Pública, v. 18, n. 6, p. 1713-1724, 2002.

SOUZA, Cezarina Maria Nobre; FREITAS, Carlos Machado. Discursos de usuários sobre uma intervenção em saneamento: uma análise na ótica da promoção da Saúde e da prevenção de doenças. Engenharia Sanitária e Ambiental, v. 14, n.1, Jan./mar. p. 59-68, 2009.

SPERLING, Tiago Lages Von; SPERLING, Marcos Von. Proposição de um sistema de indicadores de desempenho para avaliação da qualidade dos serviços de esgotamento sanitário. Engenharia Sanitaria e Ambiental, v. 18, n. 4, p. 313-322, 2013.

TEIXEIRA, Júlio César; OLIVEIRA, Guilherme Soares.; VIALI, Amanda de Melo; MUNIZ, Samuel Soares. Estudo do impacto das deficiências de saneamento básico sobre a saúde pública no Brasil no período de 2001 a 2009. Engenharia Sanitária e Ambiental, v. 19, n.1, p. 87-96, Jan./Mar. 2014.

TRABUCCHI, Mattia; NTSHOTSHO, Phumza; O'FARRELL, Patrick; COMÍN, Francisco A. Ecosystem service trends in basin-scale restoration initiatives: a review. Journal of Environmental Management, v.111, p. 18-23, 2012.

VICQ, Raphael de,; LEITE, Mariangela Garcia Praça. Avaliação da implantação de fossas sépticas na melhoria na qualidade de águas superficiais em comunidades rurais. Engenharia Sanitária e Ambiental, v. 19, n.4, p. 411-416, Out./Dez. 2014.

Duarcides Ferreira Mariosa - Possui Graduação em Ciências Sociais, Mestrado e Doutorado em Sociologia, todos cursados pela Universidade Estadual de Campinas (UNICAMP). Atualmente é Professor do Programa de Pós-Graduação em Sustentabilidade da Pontifícia Universidade Católica de Campinas (PUC-Campinas).

Samuel Carvalho De Benedicto - Possui Gradução em Ciências Físicas e Biológicas, em Biologia, em Pedagogia pela Universidade do Estado de Minas Gerais (UEMG), Graduação em Administração pela Universidade Anhanguera; Mestrado e Doutorado em Administração pela Universidade Federal de Lavras (UFLA). Atualmente é Professor do Programa de Pós-Graduação em Sustentabilidade da Pontifícia Universidade Católica de Campinas (PUC-Campinas).

Marcos Ricardo Rosa Georges - Graduação em Matemática Aplicada e Computacional, Mestrado e Doutorado em Engenharia Mecânica, todos cursados pela Universidade Estadual 
de Campinas (UNICAMP). Atualmente é Professor do Programa de Pós-Graduação em Sustentabilidade da Pontifícia Universidade Católica de Campinas (PUC-Campinas).

Cibele Roberta Sugahara - Possui Graduação em Graduação em Administração de Empresas pela Pontifícia Universidade Católica de Campinas (PUC/Campinas), Mestrado em Ciência da Informação pela Pontifícia Universidade Católica de Campinas (PUC/Campinas) e Doutorado em Ciência da Informação pela Universidade de São Paulo (USP). Atualmente é Professora do Programa de Pós-Graduação em Sustentabilidade da Pontifícia Universidade Católica de Campinas (PUC-Campinas).

Artigo recebido em: 17 de março de 2020.

Artigo aceito em: 29 de maio de 2020.

Artigo publicado em: 26 de junho de 2020. 